[38] R. A. Freeman and P. V. Kokotović, "Optimal nonlinear controllers for feedback linearizable systems," in Proc. American Contr. Conf., Seattle, WA, 1995, pp. 2722-2726.

[39] B. Wie, H. Weiss, and A. Arapostathis, "Quaternion feedback regulator for spacecraft eigenaxis rotation," J. Guidance, Contr. Dynam., vol. 12, pp. $375-380,1989$.

\section{Systems with Finite Communication Bandwidth Constraints-II: Stabilization with Limited Information Feedback}

Wing Shing Wong and Roger W. Brockett

\begin{abstract}
In this paper a new class of feedback control problems is introduced. Unlike classical models, the systems considered here have communication channel constraints. As a result, the issue of coding and communication protocol becomes an integral part of the analysis. Since these systems cannot be asymptotically stabilized if the underlying dynamics are unstable, a weaker stability concept called containability is introduced. A key result connects containability with an inequality equation involving the communication data rate and the rate of change of the state.
\end{abstract}

Index Terms - Asymptotic stability, containability, feedback control, Kraft inequality.

\section{INTRODUCTION}

In the early part of this decade several papers appeared which investigated various information related aspects of decision and control. These included work by Delchamps [4], Kabamba and Hara [6], and Williamson [10]. For example, Delchamps studied the problem of stabilizing a discrete-time linear system with quantized state feedback. Quantization is, of course, a crucial consideration, but informationally related issues involve a much wider range of questions. In a previous paper by the authors [11], a class of estimation problems with communication constraints was introduced and analyzed. It was shown, in particular, that the performance of estimation algorithms is closely related to the data rate and the time scale of the underlying dynamical system. The motivation for investigating these systems came from a variety of sources including neurobiological systems, social-economical systems, and remotely controlled systems, (see, for example, the problem of power control in wireless communication studied in [9]). This class of systems is substantially different from those studied in [4], [6], and [10] because the issues of coding, communication protocol, and delays are not only explicitly considered but actually form the focal point of the investigation. Recent papers by Borkar and Mitter [2] and Li and Wong [8] also adopt a similar perspective.

In this paper, we continue the analysis of communication constrained systems, studying the effect of the communication rate on

Manuscript received December 19, 1996. Recommended by Associate Editor, W.-B. Gong. This work was supported by a grant from the Hong Kong Research Grants Council.

W. S. Wong is with the Department of Information Engineering, Chinese University of Hong Kong, Shatin, N.T. Hong Kong.

R. W. Brockett is with the Division of Engineering and Applied Sciences, Harvard University, Cambridge, MA 02138 USA.

Publisher Item Identifier S 0018-9286(99)02118-2. a class of linear feedback control problems. The model studied here can be viewed as a variant of the classical linear feedback control problem. However, unlike the classical problem where the assumption is that the plant and the feedback controller are either colocated or they can communicate with each other over a channel with infinite capacity, the crux of the problem studied here is that the plant and the feedback controller communicate over a digital channel with finite capacity. This simple change in the basic assumption has a substantial effect on the complexity of the problem. First of all, the issue of coding and communication protocol becomes an integral part of the analysis and cannot be decoupled from the control law design. Second, there is an inherent delay in the feedback control that further complicates matters. In particular, one simple consequence is that such communication constrained systems can never be asymptotically stabilized if the uncontrolled dynamics are unstable. Instead, a weaker stability concept called containability is introduced. The concept of containability is closely related to what has been called practical stability [7]. A key result in this paper connects containability with the Kraft inequality [3] and a newly derived inequality that involves the communication data rate and the rate of change of the state.

\section{The Finite Communication Control Problem}

Consider a system with linear dynamics

$$
\left\{\begin{array}{l}
\frac{d}{d t} x(t)=A x(t)+B u(t), \quad x(0)=x_{0} \\
y(t)=C x(t)
\end{array}\right.
$$

where the state $x(t)$ is an element in $\Re^{n}, u(t)$ is a $m$-dimensional vector of control, $y(t)$ is a $p$-dimensional observation, and $A, B$, and $C$ are $n$ by $n, n$ by $m$, and $p$ by $n$ constant matrices, respectively.

The observation of $x(t), y(t)$ is transmitted to a remote decisionmaker for computing the appropriate level of feedback control. The communication channel is assumed to have a data rate of $R$ bits per second. For simplicity, we ignore the detailed implementation issues in the communication protocol and simply assume that it takes $\delta=1 / R$ s to send one bit from the plant to the controller and vice versa from the controller to the plant. Hence, if a bit is sent at time zero, it will be received at time $\delta$ at the receiver. Unlike classical models, the observed information is not transmitted continuously. Hence, we assume that $x(t)$ is sampled at time instances $\left\{r_{i}\right\}_{i=0}^{\infty}$ with $r_{0}=0$; the other sample instances will be defined later. Before an observation can be transmitted, it must be quantized and coded for the transmission. We assume that prefix codes are used so that the termination of a codeword is immediately recognizable [3]. The quantization and coding function can be symbolically represented by a function $h$ from the state space $\mathcal{R}^{p}$ to $\mathcal{B}$ where $\mathcal{B}$ stands for the set of finite length strings of symbols from a $D$-ary symbol set. $c_{i}$, the $i$ th transmitted codeword from the plant to the controller, can be represented as

$$
c_{i}=h\left(y\left(r_{i}\right)\right) \text {. }
$$

It is assumed in this paper that $h$ is a measurable function so that $h^{-1}(c)$ for any codeword $c$ is measurable.

We use variable length codewords. The codeword length function is denoted by $l$. Denote the time the $i$ th codeword $c_{i}$ is received at the feedback decision-maker by $s_{i}$. Once the coded observation is received, it is decoded and the feedback control is computed and then coded for transmission back to the plant. We assume there is no computation delay. However, there is a transmission delay due 

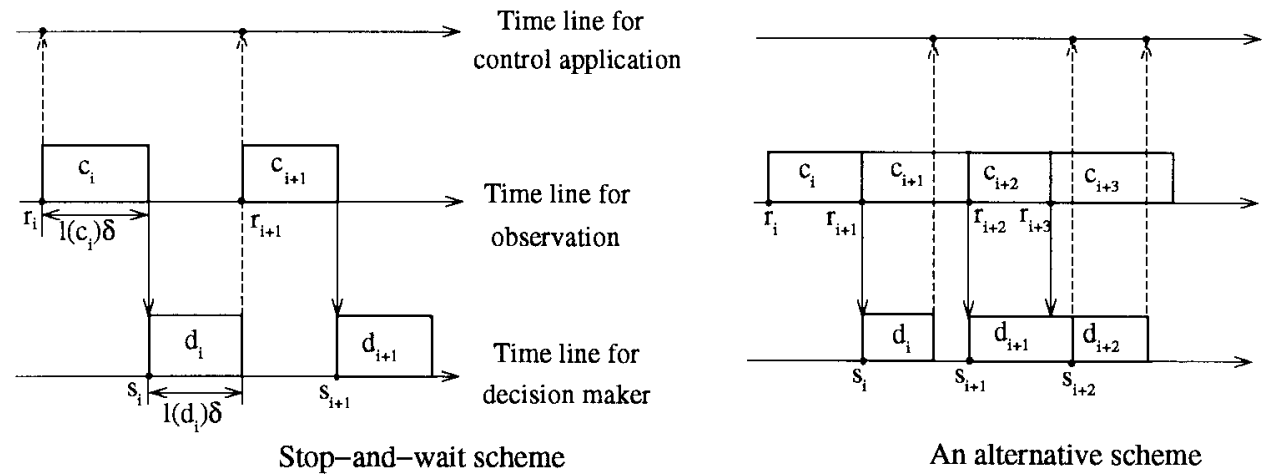

Stop-and-wait scheme

An alternative scheme

Fig. 1. Relation between $r_{i}$ and $s_{i}$.

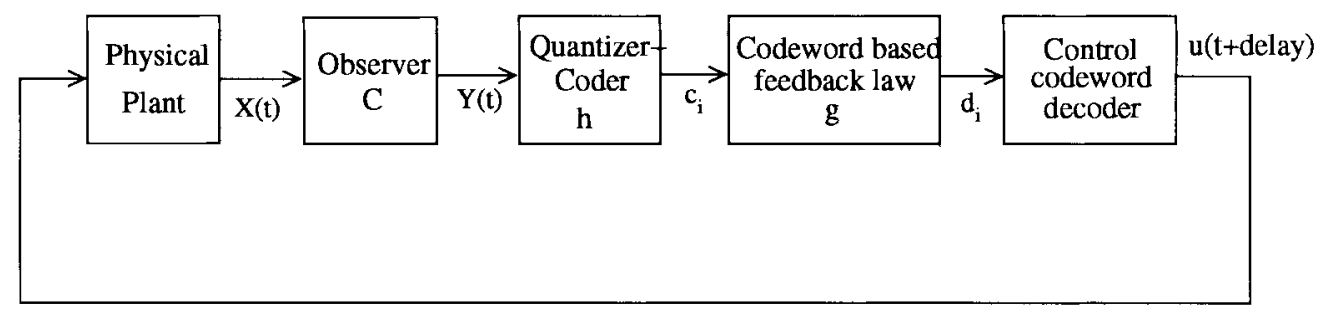

Fig. 2. Schematic of a memoryless finite communication control model.

to communication bandwidth constraints as before. The $i$ th control codeword is denoted by $d_{i}$

$$
d_{i}=g_{i}\left(c_{0}, \cdots, c_{i}\right)
$$

where $g_{i}$ is a function from $\mathcal{B}^{i+1}$ to $\mathcal{B}$.

In general, the feedback control can depend on the past history of received codewords. If the feedback law is such that the control only depends on the most recently received message we have

$$
d_{i}=g\left(c_{i}\right)
$$

with $g$ being a function from $\mathcal{B}$ to $\mathcal{B}$. This will be called a memoryless feedback law. Once the control codeword is received, it is decoded and translated into a suitable control by means of a codebook. We assume only impulse controls are used; that is, the control is in effect only for an arbitrary short duration. Let $k$ denote the mapping defined by the codebook. Then, the feedback control defined by $k\left(d_{i}\right)$ is received and applied at the plant at time $s_{i}+l\left(d_{i}\right) \delta$.

The relation between the time instants $\left\{r_{i}\right\}$ and $\left\{s_{i}\right\}$ can be defined in different ways, depending on the prearranged protocol. In this paper, a scheme which is similar to the stop-and-wait ARQ protocol [1] is assumed. Namely, after a coded observation of $x$ is sent, the next observation instant is defined to be the time after the corresponding feedback control information has been received and applied. Hence, the sequences $\left\{r_{i}\right\}$ and $\left\{s_{i}\right\}$ can be recursively defined by the following equations:

$$
\left\{\begin{array}{l}
s_{i}=r_{i}+l\left(c_{i}\right) \delta \\
r_{i+1}=s_{i}+l\left(d_{i}\right) \delta, \quad r_{0}=0 .
\end{array}\right.
$$

The relation between these time instants is illustrated in Fig. 1(a). An alternative way to define the sampling instants is to define them "back-to-back" as in Fig. 1(b). This is loosely related to the more complicated continuous ARQ protocol.
In summary, we define the memoryless finite communication control model by the following set of equations:

$$
\begin{cases}\frac{d}{d t} x(t)=A x(t)+B u(t), & x(0)=x_{0} \\ y(t)=C x(t) & \\ c_{i}=h\left(y\left(r_{i}\right)\right) & \\ d_{i}=g\left(c_{i}\right) & \\ s_{i}=r_{i}+l\left(c_{i}\right) \delta & r_{0}=0 \\ r_{i+1}=s_{i}+l\left(d_{i}\right) \delta, & \\ u\left(r_{i+1}\right)=k\left(d_{i}\right) . & \end{cases}
$$

A schematic diagram showing the relation between the various equations is shown in Fig. 2.

Notice that it is possible to combine the effect of the functions $g$ and $h$ together into one composite function. Call this composite function the coded feedback control law of the system. In theory, this coded feedback control law can be precomputed and stored at the plant. This will avoid the need of communication totally. However, in practice many systems separate the decision-maker from the plant and control it remotely for reliability and other considerations. Many neurobiological systems fit this paradigm. From an engineering perspective, if the dynamics or the control objective change from time to time it is desirable in some cases to implement simple codebooks that translate codewords into various fixed modes of control than to compute complicated algorithms to handle these changes directly at the plant.

It should be pointed out that in this model the initial control is set to zero until the first observation is obtained. We call this model an unprepared model. It is possible to consider models that have precomputed initial control. From a practical viewpoint, this imposes a severe constraint on the system because it implies the initial state and start time of the system must be known quite accurately. In this paper, we will consider only unprepared systems.

An unprepared system with an unstable dynamics cannot be made asymptotically stable by a coded feedback control law. This is due to the delay between observation instants and control time. For the 
initial $2 \delta$ or more time units, the system is essentially an uncontrolled system. It follows that we need a weaker stability condition in order to study these systems. This leads to the following notion of containability.

\section{NOTION OF CONTAINABILITY}

For the linear systems defined in this paper, we introduce the following concept of containability.

Definition 1: A finite communication control system on $\Re^{n}$ is containable if for any sphere $N$ centered at the origin there exists an open neighborhood of the origin $M$ and coding and feedback control laws such that any trajectory started in $M$ remains in $N$ for all time.

The notion of containability is closely related to the concept of practical stability and uniform practical stability [7]. In particular, the system defined by (1) is uniformly practically stable if given $(\lambda, A)$ with $0<\lambda<A,\left|x_{0}\right|<\lambda$ implies $|x(t)|<A$ for all $t \geq 0$. Hence, one of the major differences between the two concepts is that containability is not predicated on the tuple $(\lambda, A)$.

Notice that the definition of containability implies that $M$ is a subset of $N$. Suppose that coding and feedback laws, $h$ and $g$, are fixed in a finite communication control system, and define $L_{N, h, g}$ to be the maximal set such that all trajectories starting in it remain in $N$ for all time. By definition, if the system starts from a point outside of $L_{N, h, g}$, its trajectory will leave $N$ in finite time. If a system is containable, then for any sphere $N$ there exist coding and feedback laws, $h$ and $g$, that confine trajectories to $N$. We denote the set $L_{N, h, g}$ simply as $L_{N}$ if there is no confusion.

It follows from the definition of containability that there exists a set with nonzero Lebesgue measure inside $L_{N}$. Hence, the system satisfies

$$
\mu\left(L_{N}\right)>0 .
$$

A simple observation that is crucial to our subsequent argument is that the sampling instants, $\left\{r_{i}\right\}$, behave like renewal epochs in the sense stated in the following lemma.

Lemma 1: Suppose that coding rule $h$ and the memoryless feedback law $g$ are fixed. If $x(0)$ is contained in $L_{N, h, g}$, then $x\left(r_{i}\right)$ is also contained in $L_{N, h, g}$ for all positive $i$.

Proof: Suppose that $x\left(r_{i}\right)$ is outside of $L_{N, h, g}$. By the definition of $L_{N, h, g}$ such a trajectory will leave $N$ in finite time. Since the coding and feedback laws are both memoryless, the trajectory after $r_{i}$ is identical to the trajectory starting at $x\left(r_{i}\right)$ at time zero, a contradiction.

It should be emphasized that in between time instants $r_{i}$ 's, a "contained" trajectory may wander outside of $L_{N, h, g}$ but inside of $N$ as shown in Fig. 3. (Note that the trajectory starting from $x(t)$ for $r_{0}<t<r_{1}$ may be subject to a totally different set of control inputs.)

\section{NeCESSARY CONDITIONS FOR CONTAINABILITY}

In this section, a set of necessary conditions for a memoryless finite communication control system to be containable is derived. These conditions relate information theoretic characteristics with the dynamics of the system.

Let $\mathbf{K}$ be a subset of $\Re^{n}$. If matrix $A$ is $n$ by $n$ and vector $b$ is $n$-dimensional, define the set $A \mathbf{K}+b$ by

$$
A \mathbf{K}+b=\{A x+b: x \in \mathbf{K}\} .
$$

Define the subset of $\Re^{n}, \phi_{t}(\mathbf{K}, u)$ to be the set reached by a trajectory at time $t$ starting from an element in $\mathbf{K}$ when the control $u$ is applied.

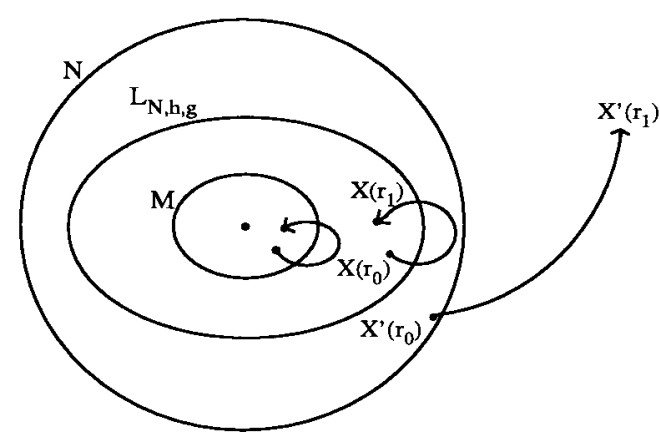

Fig. 3. All trajectories starting in $L_{N, h, g}$ stay in $N$ for all time. Those starting outside of $L_{N}, h, g$ will leave $N$ eventually.

If the control $u$ is applied over the time interval $[0, t]$ to the system defined by (1), then the terminal state at time $t$ is given by

$$
x(t)=e^{t A} x_{0}+\int_{0}^{t} e^{(t-s) A} B u(s) d s .
$$

It follows that if the initial states lie in a set $\mathbf{K}$, then

$$
\phi_{t}(\mathbf{K}, u)=e^{t A} \mathbf{K}+v(u)
$$

for some vector $v(u)$. If $\mathbf{K}$ is Lebesgue measurable, then the Lebesgue measure of $\phi_{t}(\mathbf{K}, u)$ is given by the formula

$$
\mu\left(\phi_{t}(\mathbf{K}, u)\right)=\operatorname{det}\left(e^{t A}\right) \mu(\mathbf{K})=e^{t \operatorname{tr} A} \mu(\mathbf{K}) .
$$

Denote $e^{\delta \operatorname{tr} A}$ by $\tau$.

Denote the set of $D$-ary codewords in a memoryless finite communication control system by $\mathcal{E}=\left\{e_{0}, e_{1}, \cdots\right\}$. Since the feedback law is memoryless, the coded observation $e_{i}$ will always elicit the same coded feedback response, denoted by $f_{i}$. We assume that the $f_{i}$ codewords are also based on $D$ symbols. Let $\mathcal{F}$ denote the set of $f_{i}$ codewords. Without lost of generality, we may assume the $f_{i}$ are distinct. (If observation codewords $e_{i}$ and $e_{j}$ elicit the same coded feedback response we can eliminate one of the observation codewords and use the other codeword to represent both sets of observed states.) Let $\aleph$ represent the cardinality of the set of observation codewords, which can be assumed to be identical to the cardinality of the set of feedback control codewords as previously explained.

Theorem 1: Consider a containable, memoryless finite communication control law for (1) that uses $D$-ary codewords. Let $N$ be an arbitrary sphere centered at the origin in $\Re^{n}$ and $h$ and $g$ be the corresponding coding and feedback control laws that ensure all trajectories starting from an open neighborhood of the center, $M$, remain in $N$ for all $t \geq 0$. Let $m_{i}=l\left(e_{i}\right)$ and $n_{i}=l\left(f_{i}\right)$. (Recall that $l$ is the codeword length function.) Then the following inequalities hold:

$$
\begin{aligned}
& \text { 1) } \sum_{0}^{\infty} 1 / D^{m_{i}} \leq 1 \\
& \text { 2) } \quad \sum_{0}^{\infty} 1 / D^{n_{i}} \leq 1 \\
& \text { 3) } 1 \leq \sum_{0}^{\infty} 1 / \tau^{m_{i}+n_{i}}
\end{aligned}
$$

with $\tau=e^{\delta \operatorname{tr} A}$ and $\delta$ the transmission delay.

Proof: The first two inequalities are the well-known Kraft inequality which must hold in order if it is to be possible to construct the codeword set with the prescribed codeword lengths [3]. To prove the third inequality, let $U_{i}$ be the subset of $\Re^{n}$ such that for any $x \in U_{i}$

$$
h(C(x))=e_{i}
$$


It follows that $\bigcup U_{i}=\Re^{n}$. Define $L_{N}$ as stated in the previous section. Let

$$
L_{i}=U_{i} \bigcap L_{N}
$$

It is easy to see that the $L_{i}$ 's are measurable.

If the initial point $x_{0}$ is in $L_{i}$, then at sampling time $r_{1}, x\left(r_{1}\right) \in$ $L_{N}$ as implied by the assumption that the system is containable and Lemma 1. Moreover

$$
r_{1}=\left(m_{i}+n_{i}\right) \delta .
$$

If the decoded control is denoted by $u_{1}$, then

$$
x\left(r_{1}\right)=e^{\left(m_{i}+n_{i}\right) \delta A} x_{0}+v\left(u_{1}\right)
$$

for some vector $v\left(u_{1}\right)$.

Let the set of states at time $r_{1}$ for all trajectories starting in $L_{i}$ be denoted by $M_{i}$. Then

$$
M_{i}=e^{\left(m_{i}+n_{i}\right) \delta A} L_{i}+v\left(u_{1}\right) .
$$

From the containable assumption

$$
M_{i} \subset L
$$

or

$$
e^{\left(m_{i}+n_{i}\right) \delta A} L_{i}+v\left(u_{1}\right) \subset L
$$

It follows that

$$
\mu\left(e^{\left(m_{i}+n_{i}\right) \delta A} L_{i}\right) \leq \mu(L)
$$

Hence

$$
\mu(L) \geq \tau^{\left(m_{i}+n_{i}\right)} \mu\left(L_{i}\right)
$$

Since

$$
\sum_{i}^{\aleph} \mu\left(L_{i}\right)=\mu(L)>0
$$

it follows from (20) that

$$
1 \leq \sum_{0}^{\aleph} 1 / \tau^{m_{i}+n_{i}} .
$$

One can derive the following necessary condition as a corollary of this result.

Corollary 1: If a memoryless finite communication control system uses the same set of codewords for observation and feedback control, then it is containable only if

$$
\tau^{2} \leq D
$$

Proof: If $m_{i}$ 's and $n_{i}$ 's form sequences that are related to each other by permutation of the indexes, then by the Hardy-Littlewood-Polya rearrangement theorem [5], it follows that the term $\sum_{0}^{\aleph} 1 / \tau^{m_{i}} 1 / \tau^{n_{i}}$ is maximized when $m_{i}=n_{i}$. Hence, it follows from Theorem 1 that

$$
1 \leq \sum_{i=0}^{\aleph} 1 / \tau^{2 m_{i}}=\sum_{i=0}^{\aleph} 1 / \tau^{2 n_{i}} .
$$

If $\tau^{2}>D$, then

$$
1 \leq \sum_{i=0}^{\aleph} 1 / \tau^{2 m_{i}}<\sum_{i=0}^{\aleph} 1 / D^{m_{i}} \leq 1 .
$$

A contradiction.

\section{SuFFICIENT CONDITIONS FOR CONTAINABILITY}

In this section, we will consider some sufficient conditions for systems to be containable. For simplicity, we assume from now on that $m=n=p$, that is the dimension of the control vector is same as the dimension of the observation vector, which is equal to the dimension of the state space.

Define $\tau_{\infty}$ to be the norm $\left\|e^{\delta A}\right\|_{\infty}$. That is

$$
\tau_{\infty}=\max _{\|x\|_{l \infty}=1}\left\|e^{\delta A} x\right\|_{l \infty} .
$$

The following sufficiency result applies to any dimension $n$.

Theorem 2: Consider a memoryless finite communication control system with $(A, B)$ forming a controllable pair and $C$ being invertible. Assume that binary codewords are used. Then the system is containable if

$$
\tau_{\infty}^{2^{n}+1}<2
$$

Proof: If one regards $h^{\prime}(x)=h(C(x))$ as the coding function, one can assume that $y(t)=x(t)$, to simplify the presentation.

Let $I_{n}$ be an $n$-dimensional cube with sides parallel to the axes and side length $l$. A key step in proving the theorem is to show that it is possible to construct coding and feedback control laws so that all trajectories starting in $I_{n}$ at time zero return to $I_{n}$ at $r_{1}$, the next sampling time. As observed before, since these sampling times, $\left\{r_{i}\right\}$, behave like renewal epochs, the same coding and feedback control laws can be used to guarantee that future trajectories will always return to $I_{n}$ at all sampling times, $r_{i}$.

To construct the coding and feedback laws, divide $I_{n}$ into $2^{n}$ cubes with equal volume $(l / 2)^{n}$ and label them as $L_{i}$ 's. Notice that each cube has sides of length equal to $l / 2$. We assume that the surfaces of the $L_{i}$ 's are assigned in a well-defined way so that the $L_{i}$ 's form a partition of $I_{n}$. All points observed in $L_{i}$ will be coded with the binary codeword $e_{i}$ with length $i$. Since there are $2^{n}$ subcubes, the codeword length varies from 1 to $2^{n}$. According to Kraft's lemma, such a codeword set exists. The feedback control $u_{i}$, corresponding to the codeword $c_{i}$, is coded by the codeword $e_{2^{n}-i+1}$. It follows that as shown before, if $x(0) \in L_{i}$ then $x\left(r_{1}\right)$ is contained in the set

$$
e^{\left(2^{n}+1\right) \delta A} L_{i}+v\left(u_{i}\right)
$$

for some vector $v\left(u_{i}\right)$ which is dependent on the control law $u_{i}$.

The image of $L_{i}$ at the sampling time $r_{1}$ can be contained in a cube $C$ with sides parallel to the axes and length no greater than $l / 2 \tau_{\infty}^{2^{n}+1}$. Since

$$
\tau_{\infty}^{2^{n}+1}<2
$$

it is possible to find an impulse control $u_{i}$ to move $C$ inside $I_{n}$.

To show that the system is containable in a sphere $N$, centered at the origin, construct a cube $M$ centered at the origin with sides equal to $\epsilon$ and parallel to the axes. Apply the coding and feedback control laws defined previously. At time $r_{1}$, the trajectories stay in $M$. Since there are only a finite number of possible coding and control values, $r_{1}$ can assume only a finite set of values and the trajectory from $x\left(r_{0}\right)$ to $x\left(r_{1}\right)$ is uniformly bounded for all initial points in $M$. Moreover, since the system is linear, this set can be made arbitrarily small if $M$ is small enough. So, by choosing a small enough $\epsilon$, one can ensure that all trajectories starting in $M$ will be contained in $N$ for all time.

This theorem can be easily generalized to the $D$-ary codeword cases. Note that if $A$ is the identity matrix, then $\tau=e^{\delta}=\tau_{\infty}$. The necessary condition in Corollary 1 for the binary codeword case states that

$$
e^{2 \delta} \leq 2
$$

On the other hand, the sufficient condition of Theorem 2 requires that

$$
e^{\left(2^{n}+1\right) \delta} \leq 2
$$

Hence, there is a large gap between the two conditions. It is likely that a tighter necessary condition can be derived if $n>1$. Moreover, 
for the case where $n=1$, it is possible to tighten the sufficiency condition to prove the following necessary and sufficient theorem.

Theorem 3: Consider the following memoryless finite communication system with state dimension 1 :

$$
\left\{\begin{array}{l}
\frac{d x}{d t}=a x+b u, \quad x(0)=x_{0} \\
y(t)=c x(t)
\end{array}\right.
$$

with $a>0$ and $b, c$ nonzero.

Assume that the same set of codewords are used for coding and feedback control. Then the system is containable if and only if

$$
\tau^{2} \leq D
$$

Proof: For a system with state dimension $1, \tau=\tau_{\infty}=e^{\delta}$. Hence, the necessary part follows from Corollary 1 .

For the sufficiency part, partition the interval $[0,1)$ so that there are $D-1$ first level subintervals each with length $\left(\tau^{2}-1\right) /(D-$ 1) $\left(\tau^{2}\right)$; there are $D-1$ second level subintervals each with length $\left(\left(\tau^{2}-1\right) /(D-1)\left(\tau^{2}\right)\right)\left(1 / \tau^{2}\right)$; there are $D-1$ third level subintervals each with length $\left(\left(\tau^{2}-1\right) /(D-1)\left(\tau^{2}\right)\right)\left(1 / \tau^{4}\right)$, and so on. The endpoints of these intervals are assumed to be closed on the left and open on the right. Let $1,2, \cdots, D$ denote the $D$ symbols used in the codeword. Define a coding scheme by mapping the $j$ th subintervals in the $i$ th level by a codeword with length $i$ where the first $i-1$ symbols are $D$ and the last symbol is $j$. For example, if $i=3$, then the codeword is $(D, D, j)$ for the $j$ th subinterval. Define the coding scheme for the feedback law so that if the codeword $c_{i}$ is received, the intended feedback law is coded by the same codeword. Since the system is clearly reachable, there exists feedback control for each subinterval to ensure it stays within the interval $[0,1)$ at the next sampling instant provided

$$
\tau^{2(i+1)} \frac{\tau^{2}-1}{(D-1)\left(\tau^{2}\right)} \frac{1}{\tau^{2 i}} \leq 1 .
$$

This holds if $\tau^{2} \leq D$. The rest of the argument follows as in Theorem 2.

\section{CONCLUSION}

In this paper, the issue of feedback control of a system with finite communication constraint is considered. The concept of containability is introduced and simple necessary and sufficient conditions for containability are derived. The problems introduced in this paper are relatively new and much more effort is needed in the future to provide deeper insight into this class of systems. An interesting geometric question central to the issue is the following:

Problem: Consider a unit cube or sphere in $\Re^{n}, I$. Given a function $f$ that maps $I$ into $\Re^{n}$, what are the necessary and sufficient conditions for the existence of a partition of $I$ into $I_{n}$ 's and a corresponding sequence $\left\{m_{i}\right\}$ satisfying $\sum 1 / 2^{m_{i}} \leq 1$, such that

$$
f^{m_{i}}\left(I_{n}\right)+v_{i} \subset I
$$

for some vector $v_{i}$ for all $i$ ?

The results in this paper provide only a partial answer to this question. There is a possibility that weaker sufficiency conditions can be obtained for the case when $A$ has some stable eigenvalues. Another interesting question concerns the behavior of the trajectory set defined by $x\left(r_{i}\right)$. It is likely under suitable conditions that this sequence may exhibit chaotic motion behavior.

\section{REFERENCES}

[1] D. Bertsekas and R. Gallager, Data Networks. Englewood Cliffs, NJ: Prentice-Hall, 1992.

[2] V. S. Borkar and S. K. Mitter, "LQG control with communication constraints," in Communications, Computation, Control and Signal Processing, a Tribute to Thomas Kailath, A. Paulraj, V. Roychowdhury, and C. D. Schaper, Eds. New York: Kluwer, 1997, pp. 365-373.

[3] T. M. Cover and J. A. Thomas, Elements of Information Theory. New York: Wiley-Interscience, 1991.

[4] D. F. Delchamps, "Stabilizing a linear system with quantized state feedback," IEEE Trans. Automat. Contr., vol. 35, pp. 916-924, 1990.

[5] G. H. Hardy, J. E. Littlewood, and G. Polya, Inequalities, 2nd ed. Cambridge, U.K.: Cambridge Univ. Press, 1952.

[6] P. T. Kabamba and S. Hara, "Worst-case analysis and design of sampled-data control systems," IEEE Trans. Automat. Contr., vol. 38, pp. 1337-1357, Sept. 1993.

[7] V. Lakshmikantham, S. Leela, and A. A. Martynyuk, Practical Stability of Nonlinear Systems. Singapore: World Scientific, 1990.

[8] X. Li and W. S. Wong, "State estimation with communication constraint," Syst. Contr. Lett., vol. 28, pp. 49-54, 1996.

[9] _ , "Coder-estimator sequence for power control," in Proc. Amer Control Conf., Albuquerque, NM, June 4-6, 1997, pp. 220-221.

[10] D. Williamson, "Finite wordlength design of digital Kalman filters for state estimation," IEEE Trans. Automat. Contr., vol. 30, pp. 930-939, 1990.

[11] W. S. Wong and R. W. Brockett, "Systems with finite communication bandwidth constraints-I: State estimation problems," IEEE Trans. Automat. Contr., vol. 42, pp. 1294-1298, Sept. 1997.

\section{Sufficient LMI Conditions for Output Feedback Control Problems}

\author{
César A. R. Crusius and Alexandre Trofino
}

\begin{abstract}
In this paper the authors present linear matrix inequality (LMI) conditions for output feedback control problems. The results are based on sufficient conditions because they are dependent on the particular state-space representation used for describing the system. Nevertheless, the conditions are not sensitive to a certain class of state-space transformations, and if the control problem is feasible then there exists some state-space transformation leading the conditions to be necessary and sufficient for the problem. The authors approach can be used for designing decentralized controllers and is easily extended to $\mathcal{H}_{2}, \mathcal{H}_{\infty}$ and mixed $\mathcal{H}_{2} / \mathcal{H}_{\infty}$ problems via standard LMI techniques. The continuousand discrete-time cases are considered and numerical examples are given to illustrate the results.
\end{abstract}

Index Terms-LMI, robustness, static output feedback.

\section{INTRODUCTION}

The static output feedback stabilization is among the most important control problems for which a complete solution is not available yet. During the last decades various approaches have been proposed to deal with the problem [1]-[9].

Manuscript received September 16, 1996; revised June 30, 1997. Recommended by Associate Editor, M. A. Dahleh. This work was supported in part by $\mathrm{CNPq}$, Brazil.

C. A. R. Crusius is with Stanford University, Stanford, CA 94305 USA.

A. Trofino is with the Department of Systems and Automation, Federal University of Santa Catarina, cep 88040-900 Florianópolis-SC Brazil (e-mail: trofino@das.ufsc.br).

Publisher Item Identifier S 0018-9286(99)02119-4. 